\title{
Research on Management System of Mold Manufacturing Enterprise Based on RFID Technology
}

\author{
Bin Wang ${ }^{1}$, Defang Liu ${ }^{1}$ and Mingkun $\mathrm{Ji}^{1,2}$ \\ ${ }^{1}$ Yancheng Institute of Technology, School of Mechanical Engineering, 224051 Yancheng, China \\ ${ }^{2}$ Anhui University of Science and Technology, School of Mechanical Engineering, 232001 Huainan, China
}

\begin{abstract}
To promote manufacturing management efficiency and decrease error rate in production process, this paper proposed a management system framework of mold manufacturing enterprises based on RFID technology. The system consists of four subsystems, which are warehouse management, production management, distribution and customer. The structure of each subsystem is presented in detail based on the analysis of overall design process of mold management system. Finally, a testing table of RFID-based mold manufacturing system was established to illustrate the feasibility of the system.
\end{abstract}

\section{Introduction}

In recent years, Radio Frequency Identification (RFID) has been widely used in product manufacturing field as an automatic identification technology, which depends on remotely storing and retrieving data based on tags and readers $[1,2]$. Many manufacturing enterprises have already followed the trend of the next industrial revolution under the heading of Industrie 4.0 by investing in hardware, software, and networking systems to build the Internet of Things (IoT) [3, 4]. Reyes et al. [5] identified the determinants of RFID adoption stage and explored the perceived benefits from RFID adoption and found that RFID adoption had a significant benefits to perceived customer service and productively.

To gain insights for the factors that influence the RFID adoption, Bhattacharya [6] conducted a Delphibased research to explore opinions of experts. Munilla et al. [7] had shown that protocol falls short of its security objectives, and describe attacks that allow: (a) an eavesdropper to trace a tag, (b) the previous owner to obtain the private information that the tag shares with the new owner, and (c) an adversary that has access to the data stored on a tag to link this tag to previous interrogations (violating forward-secrecy) and had analysed the security proof and found that while the first two cases can be addressed with a more careful design, strong privacy remains an open problem for lightweight RFID applications. Tsai et al. [8] proposed a protocol named as Smart Trend-Traversal (STT) to simplify the collecting process of tag IDs, which is regarded as a timeconsuming process for most manufacturing enterprises.

To develop single node multi-parameter sensing chipless RFID sensors in versatile RF sensing applications, Amin et al. [9] proposed a novel chipless
RFID sensor platform for wireless sensing of tagged objects, which need not require any on-board micro-chip and power source. Xie et al. [10] focused on RFID data management and described the research work on algorithm, protocol and performance evaluation. In his work, he introduced the research progress in RFID with anti-collision algorithm, authentication and privacy protection protocols. Zhang et al. [11] combined IOT operation principle and working mechanism with EPC global standard and established a structural model of IOT and gave the aware-layer, network-layer and applicationlayer works in this model.

Many scholars have researched on the RFID-based manufacturing management system and achieved successes in this field. For example, Zhang et al. [12] proposed a new job-shop real-time data collecting system based on the analysis of the application demands of RFID technology in manufacturing process. His system combined multi-threads technology and database technology, which performed the functions of the realtime monitoring, the timely transfer of the materials and reasonable scheduling for production plan. Staring from the RFID application requirements analysis of enterprise management, Ni et al. [13] established an automobile manufacturing line vertically distributed application model system framework and gave the system process of each function modules, which finally was testified solving data loss and omissions in information collection and the low efficiency caused by artificial operation in the production process. In order to provide reference for RFID system and promote the optimization of manufacturing system used in discrete manufacturing enterprises, Li et al. [14] analysed RFID adoption process for discrete manufacturing enterprises and summarized adoption influencing factors. The case of automobile 
manufacturing enterprise in Chongqing was employed to testify the feasibility and effectiveness of the operation mode.

Above research achievements have greatly improved the development of RFID-based manufacturing management. While mold manufacturing enterprises has their own speciality, such multi-variety, multi-process, and small amount, a customized IoT-based management system should be studied and applied in mold manufacturing field. The aim of this study is to improve the management efficiency and decrease the error ratio in mold manufacturing process.

\section{Overall design process of loT-based mold management system}

A complete mold manufacturing system includes 4 modules such as warehouse management, production, distribution and customer communication. Fig.1 illustrates the complete IoT system of mold manufacturing.

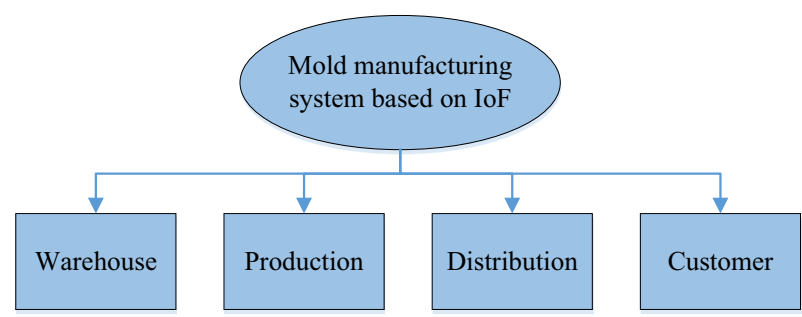

Figure 1. Main modules of mold manufacturing system based on IoF.

\subsection{Subsystem of warehouse management}

When a mold manufacturing enterprise has received an order, the subsystem of warehouse will automatically inquire the category and quantity of the raw materials, cost of manufacturing, and period of production that will be needed in this production process. The subsystem of warehouse will output an appraisement result for the possible profit of the manufacturing order, and send it managers for making a decision whether the order will be accepted. The structure of the subsystem of warehouse management is illustrated in Fig.2.

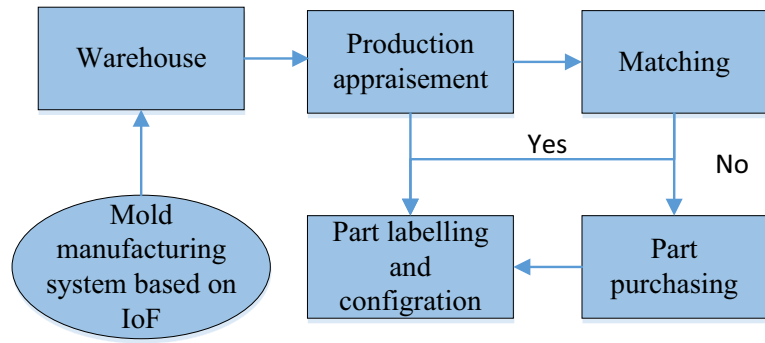

Figure 2. Subsystem of warehouse management.

If the manufacturing order is accepted, the purchasing department will make a further assessment on the required raw materials based on the output results of the subsystem of warehouse. After consulting with part suppliers, the purchasing department will submit the data of names of part suppliers, costs and types of part purchasing, quantity needed to the mold manufacturing system. The design department will design the mold meeting the requirements of the order. The overall 3D model and 2D detail drawing will be finished and submitted to the system by the design department for the reuse of other manufacturing relative departments. For example, the process department will make out a detail manufacturing process of the mold product according the 3D and 2D models and send the manufacturing tasks to the manufacturing plants via the mold manufacturing system.

\subsection{Subsystem of production management}

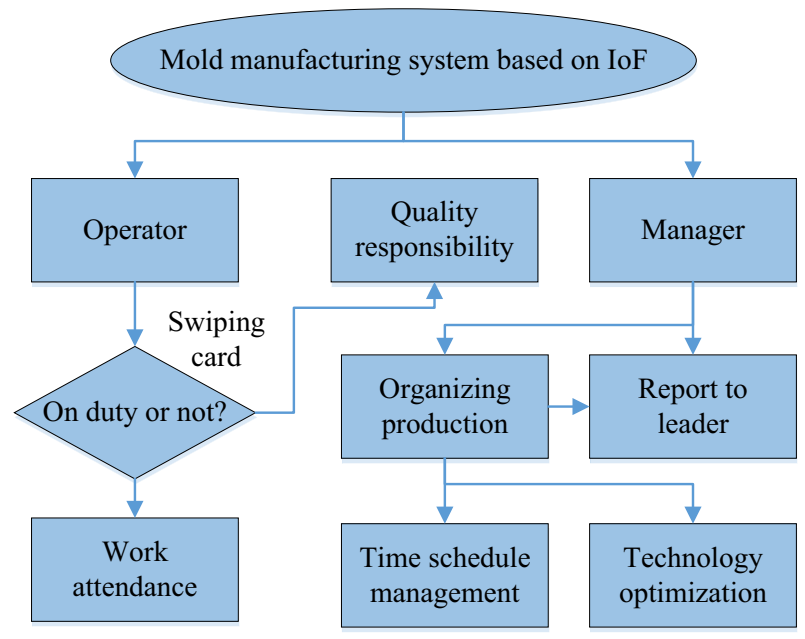

Figure 3. Information flow in production subsystem.

RFID technology employed on manufacturing process helps provide the information, performance and using states of the manufacturing equipment. With RFID, the effectiveness of overall equipment is greatly improved. There are much data needed to be collected by RFID during the mold manufacturing process, such as punching times, temperature, pressure, working condition, noise, speed, weight, and lubricating status. These data are very important for monitoring and predicting the working condition of the mold manufacturing equipment. Through punching time, we can easily deduce the next time for mold maintenance and when a new mold should be prepared in advance to keep the continuity of the production. The temperature data collected during the initial, processing, and finished time of manufacturing process can be effectively used to keep the quality the products, because product quality always has a close relationship with temperature. Pressure data collected are also used to control quality of the manufacturing process. Noise is monitored in real time to keep the safety of operators of the manufacturing equipment. The checking data of casual samples are transmitted to production subsystem and compared with normal data every a fixed span of time. If the dimensions of samples do not in the range of normal size, the mold manufacturing equipment will be forced to stop for an overhaul. Two kinds of persons are involved in the production subsystem, one is 
the direct operator of mold manufacturing equipments, and the other is the managers of manufacturing plants. The information flow between the two kinds of persons and subsystem is illustrated in Fig.3.

All kinds of sensors are employed in the process of manufacturing to monitor the production state on the site. Through sensors, the production data of temperature, pressure, noise etc. are collected and saved in the system for production management. After the manufacturing of molds, the data of assembling and testing are collected and stored in the system base for future's reuse.

\subsection{Subsystem of distribution}

RFID technology has been widely used in distribution field. In a mold manufacturing enterprise, the RFID labels are automatically input basic production information, which mainly includes delivery time, production process, number of product, and relative equipment.

The key of solution of mold product distribution is allocation and traceability of the products. The subsystem of product distribution ensure the parts to be sent to the right person. And some troubles such as parts lost and wrong distribution are avoided as well as other hardworking such as parts selection, and checking.

In RFID distribution system, each task needing to be finished should depends on high-efficient computers, which include the support of hardware, software, and database. To realize automatic tracing on materials, each cycle of RFID system should be equipped with an advanced computer, and the security of the system should also be ensured. A software system is more complicated one, which should own the abilities to identify, store and process the information collected by readers and to compare the newly collected information with stored data in the database. A powerful database is the basis of a RFID system, which can support the work of each cycle and improve the efficiency and accuracy of the system.

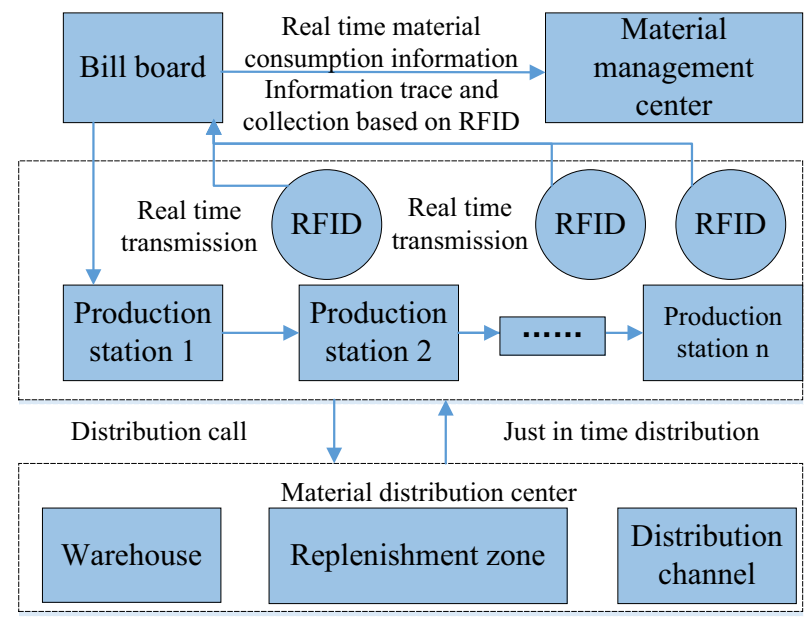

Figure 4. Information flow in production subsystem.

As shown in Fig.4, RFID is used in each production station to collect the material consumption information data, and the material distribution center acquire the real time material consumption data and prepare the materials for next distribution in advance. Thus the continuity of the production is ensured by the subsystem. Storekeepers are asked to label the RFID chips with some basic information of mold materials that will be sent the each working station. The basic information includes delivery time, production process, and product number. A GPRS location system will be encapsulated in the package of the mold. During the process of delivery, the managers can scan the transmission condition in real time to ensure the security the products.

\subsection{Subsystem of customer}

When the product is sold to customers, customer can inquire the whole manufacturing process of the product by entering the ID of RFID label. Thus the information of production, delivery position, and predicted time of arrival of the mold can be easily acquired by customers. If the some information need to be changed, customer may communicate with mold manufacturing enterprise via the mold management platform to decrease economic loss on both sides. Through the system, customers can feedback their requirements to the manufacturing enterprises in time and improve the efficiency of cooperation between them.

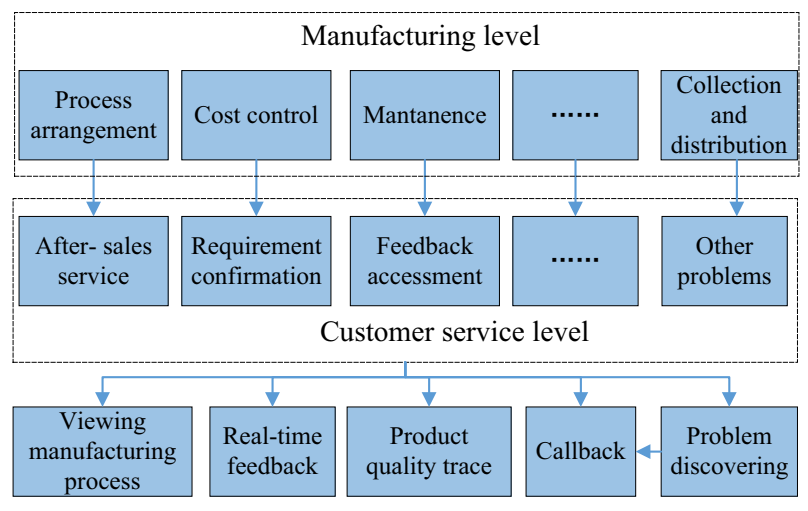

Figure 5. Information flow of customer subsystem.

From Fig.5, we can find that the information of each behaviour of customers will be collected and feedback to Cthe mold manufacturing process, which means any mold product is a comprehensive product joint-designed by customers. The subsystem of customer permits customers participating the whole manufacturing process of mold products, and thus the qualities that meets the requirements of customers are ensured.

\section{Example analysis}

An example of RFID based mold manufacturing process is employed to illustrate the working principle provided in this paper. The hardware of the system is illustrated in Fig.6, which is mainly made up of controller, mold, data displaying screen, power supply, time configurator, working table, etc.

During the product manufacturing process, terrace die are mainly relied to punch the part and form the final 
product, which is always doing telescopic movement. A controller is a main component which are used to ensure moving back and forth freely of terrace die. A circular Hall sensor is fixed on the head zone of the mold. When the mold is moving back and forth, the Hall sensor acts accordingly and transfers the collected information to liquid crystal display, which shows the current acting times in real time. Each action of mold will lead to an automatic time counting of the system, which will be shown on the LCD screen. In the hardware system, red lamp is indicator light of power supply, and green lamp is the indicator light of working condition. RFID electric labels are assembled inside the data screen mould, which can express the uniqueness of the mold. Through the unique number of the RFID label, the effective information of the mold storing in the database of the mold management system can be easily inquired. After careful processing, the data of mold manufacturing can help managers of enterprise realize information adjustment and control in real time, and achieve optimization of working efficiency of manufacturing process of the enterprise.

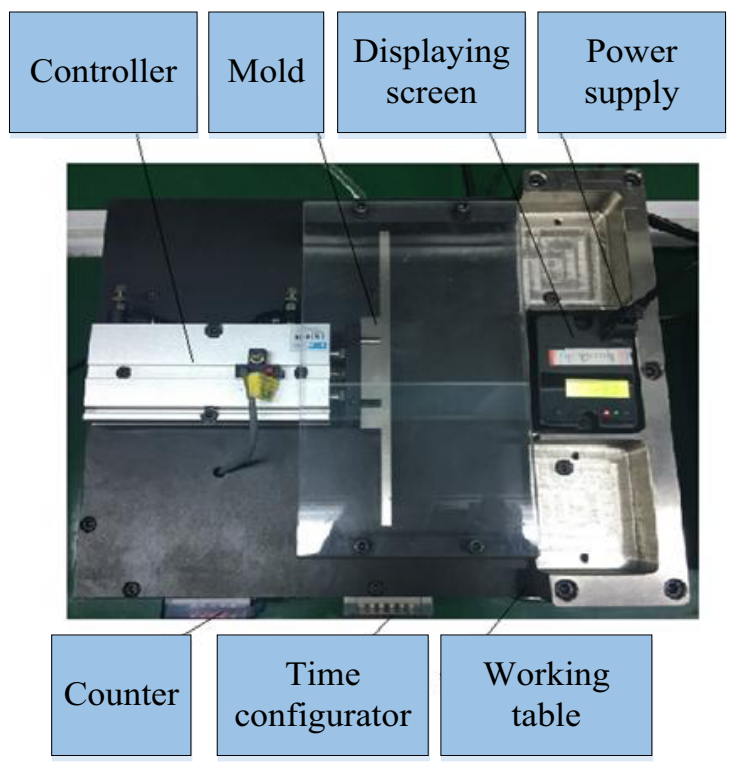

Figure 5. Testing table of RFID-based mold manufacturing system.

\section{Conclusions}

Mold enterprises are typical order-oriented discrete manufacturing enterprises. Traditional mold management mode cannot meet the requirements of practical operation because of random order and production process certainty. The research achievement of this paper helps promote the transformation and escalation of traditional mold manufacturing enterprises. RFID technology is an effective way to solve real manufacturing problems of mold factory, such as large amount of production data, frequent turnover of employees, incomplete managing records, and communication shortage among different departments, etc. RFID technology effectively accelerates the revolution of mold manufacturing pattern and promotes informatization and intellectualization of mold manufacturing enterprises. The work of this paper will display remarkable instruction for traditional mold manufacturing enterprises.

\section{Acknowledgements}

The authors gratefully acknowledge the financial support of the National Key Technology Support Program of China under Grant No. 2014BAF07B01.

\section{References}

1. X. Song, X. Li, W. Tang, W. Zhang. Information Fusion, 31 (2016), pp. 76-86.

2. S.H. Cho, K.H. Kim, S. Hong, J. Comput. Sci. Technol., 29 (1) (2014), pp. 4-20.

3. D.M. Segura Velandia, N. Kaur, W.G. Whittow, P.P. Conway, A.A. West. Robotics and ComputerIntegrated Manufacturing, 41 (2016), pp. 66-77.

4. H. Kagermann, W. Wahlster, J. Helbig, Recommendations for implementing the strategic initiative INDUSTRIE 4.0 Final report of the Industrie 4.0 Working Group, 2013.

5. P.M. Reyes, S.H. Li, J.K. Visich, European Journal of Operational Research, 254 (3) (2016), pp.801-812.

6. M. Bhattacharya, Business Process Management, 21 (3) (2015), pp.517-540.

7. J. Munilla, M. Burmester, A. Peinado, Computer Communications, 88 (15) (2016), pp.84-88.

8. S.C. Tsai, Y.M. Hu, C.H. Chai, J.S. Li, Computer Communications, 88 (15) (2016), pp.73-83.

9. E.M. Amin, N.C. Karmakar, B.W. Jensen, Sensors and Actuators A: Physical, 17 (7) (2016) (accepted) DOI:http://dx.doi.org/doi:10.1016/j.sna.2016.06.014

10. L. Xie, Y.F. Yin, X. Chen, S.L. Lu, D.X. Chen, Chinese Journal of Computers, 36 (3) (2013), pp.457-470.

11. H.D. Zhang, L. Zhu, Computer Technology and Development, 21 (5) (2011), pp.56-59.

12. Y.Y. Zhang, J. Chen, Journal of Jiangnan University (Natural Science Edition), 12 (1) (2013) pp.54-58.

13. L. Ni, H. Zhong, C. Duan, Computer Engineering, 38 (4) (2012), pp.224-226.

14. W.C. Li, Y. Jing, X. Wang, Computer Integrated Manufacturing Systems, 19 (7) (2013), pp.16841691. 